\title{
A direct boundary element approach for the numerical simulation of finite mobility ratio immiscible displacement in a Hele-Shaw cell
}

\author{
S. Jackson, D. Stevens, H. Power \& D. Giddings \\ Faculty of Engineering, Division of Energy and Sustainability, \\ University of Nottingham, UK
}

\begin{abstract}
In this work, the interaction between two immiscible fluids with a finite mobility is investigated numerically in a Hele-Shaw cell, simulating conditions found during the injection and lateral spreading of supercritical $\mathrm{CO}_{2}$ in a deep subsurface aquifer.

A two-phase numerical method is presented that uses a direct boundary element approach to compute the normal velocity at the interface between two fluids in a 2D Hele-Shaw cell, through the evaluation of a hypersingular integral. The resulting second kind Fredholm equation is solved numerically using a truncated convergent Neumann series.

Utilising cubic B-Spline surface geometry and function interpolation, the numerical scheme exhibits 6th order spatial convergence and a computational cost that scales with $O\left(N^{2}\right)$. This allows the long term non-linear dynamics of a growing $\mathrm{CO}_{2}$-brine interface to be explored accurately and efficiently, revealing large differences with previous single-phase models and interface capturing techniques.

Keywords: boundary element method, viscous fingering, finite mobility ratio, HeleShaw flow, hypersingular integral, interface tracking.
\end{abstract}

\section{Introduction}

Viscous fingering occurs during the displacement of a high viscosity fluid by a low viscosity fluid, in which interfacial instabilities may arise and subsequently evolve to form complex interface topologies. Perturbations greater than a certain 
wavelength create instabilities along the fluid interface and promote the growth of long fingers which penetrate into the more viscous fluid.

Since the work of Saffman and Taylor in 1958 [1], there has been extensive research on viscous fingering occurring in Hele-Shaw cells, where the fluid flows between two thinly separated plates. The mobility of a fluid within a Hele-Shaw cell is defined by the cell separation and the viscosity, giving rise to an intrinsic permeability, analogous to that in porous media flows.

Immiscible displacement is characterised by a large mixing time compared to flow time [2], with each fluid region having a constant density and viscosity, leading to discontinuous properties across the sharp interface. One such flow, and the motivation behind the current work, is the injection of supercritical $\mathrm{CO}_{2}$ into deep subsurface aquifers containing brine. Here, the injection process is immiscible, with the mobility ratio between the fluids typically of order 10 , involving high characteristic capillary numbers.

Most previous work in the literature has focussed on flow regimes where the mobility ratio of the fluids is typically very large, such as gas-oil injection occurring in enhanced oil recovery. Therefore, most numerical approaches consider only the external fluid, with an injected fluid of negligible viscosity, resulting in a single-phase model $[3,4]$.

Boundary element methods (BEMs) reduce the dimensionality of the problem by one and provide accurate representation of the surface, explicitly tracking it through time. In addition to BEMs for single-phase flows, BEMs have also been applied for two-phase flows, where the viscosity of both fluids is considered, resulting in a finite mobility ratio. These methods typically solve immiscible displacement between fluids with high mobility ratio, effectively reducing the problem to a single-phase approach $[3,5,6]$.

Experimental results from [7] and [8], along with numerical results from [9] and [10] suggest that the basic fingering mechanisms such as shielding, spreading and tip-splitting that occur in low mobility ratio flows are vastly different to those in single-phase (or very high mobility ratio) flows. Due to the fingers possessing significant momentum compared to those in single-phase models, finger interaction becomes much more prominent and the resulting competition can lead to coalescence and breaking [10].

To study the interaction processes and the long term evolution of low mobility ratio flows, a two-phase model is developed based on a direct boundary element approach first presented in [11]. The two-phase direct boundary element formulation proposed by Power and Wrobel [11] has not been previously implemented in the literature. Previous work has focused on indirect methods with constant boundary elements, or single-phase approaches $[11,12]$. In the proposed method, the hypersingular integral arising from the single integral equation is evaluated explicitly, resulting in a second kind Fredholm equation, which can be solved using a truncated convergent Neumann series. The resulting numerical method allows the effective modelling of a moving supercritical $\mathrm{CO}_{2}$-Brine interface in a Hele-Shaw cell, using physically realistic mobility ratios. 


\section{Mathematical formulation}

For the flow between two thinly separated plates in a Hele-Shaw cell, the depth averaged pressure and two dimensional velocity can be expressed through Darcy's law:

$$
\begin{gathered}
\vec{u}=-M \nabla P \\
\nabla \cdot \vec{u}=0
\end{gathered}
$$

Here, $M$ is the fluid mobility, which is related to the plate separation, $\mathrm{b}$, and the fluid viscosity, $\mu$ by:

$$
M=\frac{b^{2}}{12 \mu}
$$

The $b^{2} / 12$ term in the mobility ratio refers to the intrinsic permeability of the HeleShaw cell. With a constant viscosity in each of the two fluid regions, equations (1) and (2) can be reduced to laplace's equation:

$$
\nabla^{2} P^{i}(x)=0 \quad \text { for every } x \in \Omega_{i}, \quad i=1,2
$$

In the problem domain, low viscosity fluid (such as $\mathrm{CO}_{2}$ ) occupies the inner region of the bubble, $\Omega_{1}$. The region external to the bubble, $\Omega_{2}$, is filled with a high viscosity fluid such as brine. To form a boundary integral equation, the pressure field can be represented as a sum of the pressures due to an injection potential source, $Q$, and a perturbation term, $\phi_{i}$ :

$$
P^{i}(x)=\phi_{i}-\frac{Q}{2 \pi M_{i}} \ln \left(\frac{r}{a}\right) \quad \text { for } x \in \Omega_{i}
$$

In equation (5), $r$ is the radial distance from the collocation point, $x$, to the source point located inside the injected bubble and $a$ is the initial unperturbed radius of the injected fluid. At a boundary point, $\xi$ on the fluid interface, $S$, between $\Omega_{1}$ and $\Omega_{2}$, there are two matching conditions that must be met by the advancing interface. Firstly, continuity of normal velocities:

$$
q=M_{1} \frac{\partial \phi_{1}}{\partial n}=M_{2} \frac{\partial \phi_{2}}{\partial n}
$$

Secondly, the pressure jump across the interface due to the surface tension, $\gamma$ :

$$
\phi_{1}-\phi_{2}=\gamma\left(\frac{2}{b}+k(\xi)\right)+\frac{Q}{2 \pi} \ln \left(\frac{r(\xi)}{a}\right)\left(\frac{M_{2}-M_{1}}{M_{2} M_{1}}\right)=\left(M_{1}+M_{2}\right) f(\xi)
$$

In equation (7), the contact angle of the meniscus has been assumed to be zero. The signed curvature, $k(\xi)$ is considered a continuous function on the interface surface. Following from the pressure field representation in Equation (5), the 
perturbed pressures, $\phi_{1}$ and $\phi_{2}$ can be expressed in terms of their corresponding Green's formulae at the fluid interface [13] using the two dimensional fundamental solution, $\phi^{*}$.

$$
\begin{gathered}
\int_{S \infty} \phi^{*}(\xi, y) \frac{\partial \phi_{2}(y)}{\partial n_{y}} d S_{y}-\int_{S \infty} \phi_{2}(y) \frac{\partial \phi^{*}(\xi, y)}{\partial n_{y}} d S_{y} \\
+\int_{s} \phi_{2}(y) \frac{\partial \phi^{*}(\xi, y)}{\partial n_{y}} d S_{y}-\int_{s} \phi^{*}(\xi, y) \frac{\partial \phi_{2}(y)}{\partial n_{y}} d S_{y}=\frac{1}{2} \phi_{2}(\xi) \\
\int_{s} \phi_{1}(y) \frac{\partial \phi^{*}(\xi, y)}{\partial n_{y}} d S_{y}-\int_{s} \frac{\partial \phi_{1}(y)}{\partial n_{y}} \phi^{*}(\xi, y) d S_{y}=-\frac{1}{2} \phi_{1}(\xi)
\end{gathered}
$$

The external boundary at infinity in equation (8) can be evaluated asymptotically, considering the perturbation flux to approach zero as the radial distance from the source approaches infinity [14]. To combine the interior and exterior boundary integral equations (8) and (9) to produce a two-phase model applicable in both domains, the limiting value of the normal derivatives must be taken. Subtracting the resulting equations and using the matching conditions (6) and (7), the following second kind Fredholm integral equation can be formed [11].

$$
-\frac{1}{2} q(\xi)+\left(\frac{1-\beta}{\beta+1}\right) \int_{s} K(y, \xi) q(y) d S_{y}=g(\xi)
$$

Here, $\beta$ is the ratio of mobilities between the two fluids, $M_{1} / M_{2}$. The regular kernel, $K(y, \xi)$ in equation (10) is the normal derivative of the 2-D fundamental solution with respect to $\xi$. The non-homogeneous boundary term is given by the following hypersingular integral.

$$
g(\xi)=M_{1} M_{2} \int_{s} f(y) \frac{\partial K(y, \xi)}{\partial n_{y}} d S_{y}=M_{1} M_{2} \int_{s} V_{i}(\xi, y) N^{a}(\xi) J_{m} d \xi
$$

The second kind Fredholm equation in (10) permits an analytical Neumann series solution (for more details see [11]). Equation (10) can be solved using a convergent series for $q$, as long as $0 \leq \beta<\infty$ [11]. The series can be simplified by taking $\lambda=\frac{(\beta-1)}{(1-\beta)}$, using a discrete number of terms, $m$, to truncate the solution of equation (10).

$$
q(\xi)=q_{0}(\xi)+\lambda q_{1}(\xi)+\cdots+\lambda^{m} q_{m}(\xi)
$$

Successive terms in the series (12 are calculated recursively using equation (10). After convergence of the series, the movement of the interface is calculated via a forward Euler time stepping approach, where $\Delta L_{n}(\xi)$ represents the distance moved by a boundary point in a single time-step:

$$
\frac{\Delta L_{n}(\xi)}{\Delta t}=q(\xi)+\frac{Q x_{i}(\xi) n_{i}(\xi)}{2 \pi r^{2}}
$$

As the surface grows according to equation (13), the number of nodal points on the bubble boundary are adaptively increased to maintain a target element size. Cubic 
B-Splines are used for geometrical discretisation and function interpolation, due to the highly curved surfaces that require accurate representation [15].

The hypersingular integral in (11) is treated using a semi-analytical approach implemented in [16]. The hypersingular integral in equation (11) becomes an issue when the field points of integration, $y$, lie close to a collocation point, $\xi$. This is most significant on elements that coincide with the collocation point. To evaluate the hypersingular integration, firstly, the hypersingular kernel is expanded in terms of a Laurent power series about a hypersingular point, $\eta[16]$.

$$
V_{i}(\xi, y) N^{a}(\xi) J_{m}=F_{i}(\eta, y)=\frac{F_{-2}(\eta)}{(y-\eta)^{2}}+\frac{F_{-1}(\eta)}{y-\eta}+O(1)
$$

The $F_{-2}$ and $F_{-1}$ terms depend only on the derivatives of the B-spline shape functions, $N^{a}$. By introducing the above power series into the hypersingular boundary integral equation (11), the limits may be evaluated analytically in order to remove unbounded terms. This results in a regular integral and analytical expression.

$$
\begin{aligned}
g(\xi) & =M_{1} M_{2} \sum_{m=1}^{2}\left(\int_{0}^{1}\left[F^{m}(\eta, y)-\left(\frac{F_{-2}^{m}(\eta)}{(y-\eta)^{2}}+\frac{F_{-1}^{m}(\eta)}{y-\eta}\right)\right] d y\right. \\
& \left.+F_{-1}^{m}(\eta) \ln \left|\frac{1}{\beta_{m}(\eta)}\right| \operatorname{sgn}(y-\eta)-F_{-2}^{m}(\eta)\left[\operatorname{sgn}(y-\eta) \frac{\gamma_{m}(\eta)}{\beta_{m}^{2}(\eta)}+1\right]\right)
\end{aligned}
$$

In equation (15), the $\beta_{m}$ and $\gamma_{m}$ terms account for any possible distortion from an asymmetric neighbourhood around the hypersingular point [16].

\section{Numerical performance}

Here we examine the numerical performance of the two-phase model using various small scale simulations. The computational cost of the scheme scales with $m N^{2}$, where $m$ is the number of terms in the convergent series, and $N$ is the number of boundary elements. Therefore, for a fixed $m$ the scheme will exhibit second order scaling. This is much better than direct solvers for the corresponding matrix system (typically LU decomposition), which exhibit cubic scaling.

The number of terms used in the convergent series plays an important role in the accuracy and speed of solution. Figure 1 shows that as the mobility ratio of the two fluids becomes larger, the number of terms required by the convergent series to reach a desired error increases. This is because the value of $\lambda$ approaches -1 , and successive terms in the convergent series do not decay as rapidly. When $\lambda=-1$ there is no unique solution to equation (10), due to a singular value in the corresponding spectrum of the integral operator.

To investigate mesh and time independence, the model was tested under various capillary numbers, which describe the ratio of viscous driving forces to surface 


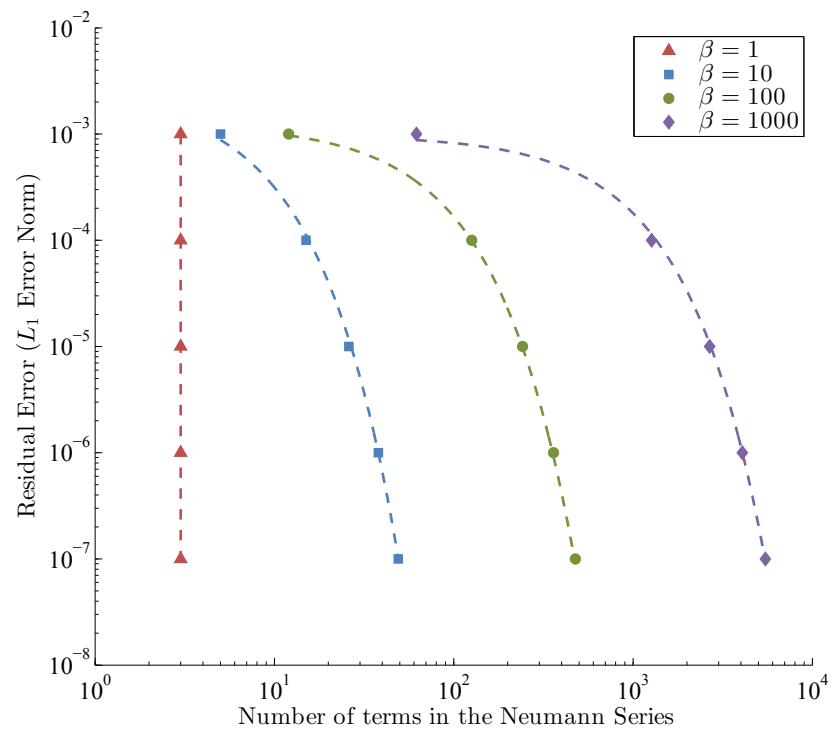

Figure 1: Residual error convergence with number of terms in the convergent series and varying mobility ratio.

tension forces. Due to the low mobility ratio being investigated, and the radial set-up of the Hele-Shaw injection, the capillary number must be modified to adequately describe the flow regime:

$$
C a^{\prime}=\frac{\mu_{2} Q}{2 \pi a \gamma}\left(\frac{a}{b}\right)^{2}=\frac{a Q}{24 \pi \gamma M_{2}}
$$

The modified capillary number (16) together with the mobility ratio uniquely describe radial Hele-shaw flow. In figure 2, the spatial convergence of the solutions can be seen. Spatially, the solution converges very quickly; for large $\Delta x$ roughly 6th order is observed. This excellent spatial convergence means that relatively few elements can be used, with very high accuracies being achieved. Temporally the solutions converge linearly, as expected from the forward Euler time stepping scheme.

\section{Mobility ratio effects}

In carbon sequestration, the $\mathrm{CO}_{2}$ injection is performed supercritically, with the mobility ratio between supercritical $\mathrm{CO}_{2}$ and brine of the order 10-30 [17]. The two-phase method presented here allows the efficient solution of such flow regimes and can be used to characterise the transition through a range of finite mobility ratios. Two finite mobility ratios are presented in figure 3, in which $C a^{\prime}=75$. 


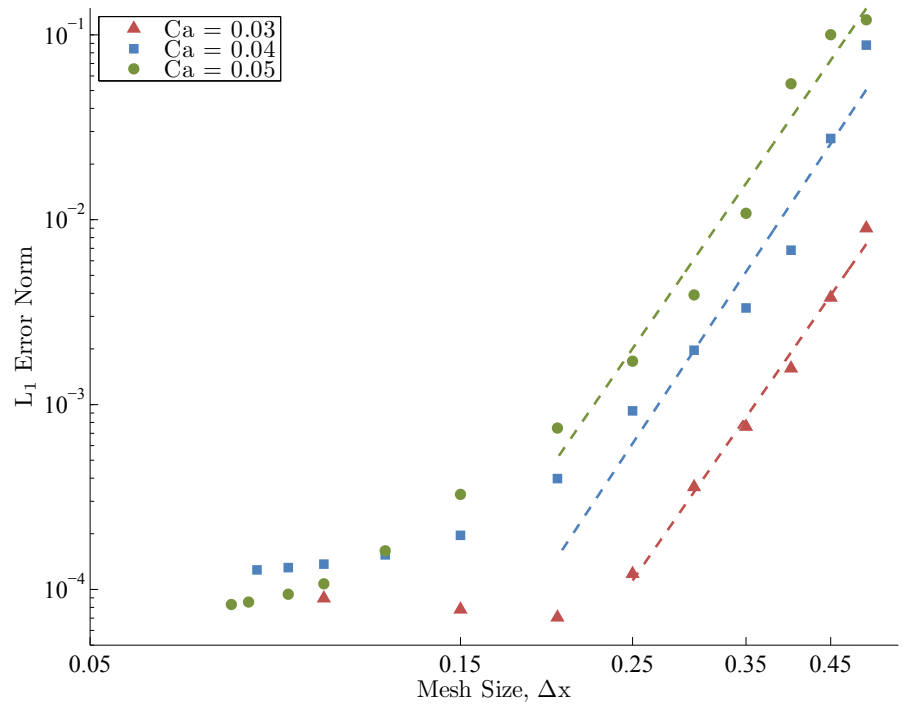

Figure 2: Mesh independence study. $L_{1}$ error norm quoted is between the numerical solution and a mesh independent case.

In the $\beta=10$ case, the bases of the fingers continue to grow with time. However, in the $\beta=1000$ case, the finger bases effectively become stagnation points, where the interface velocity at the base drops to near zero. This characteristic is a well known feature of high mobility ratio injection, causing highly convoluted surfaces and a much lower swept volume of the higher viscosity fluid. A consequence of the slowed base evolution and quickly growing primary fingers is that competing fingers' growth is hindered by the larger primary fingers and shielding occurs [2].

However, in the low mobility ratio regime, the growing finger bases allow secondary growing fingers to be fed by fluid, meaning that they can possess significant momentum. Shielding between competing fingers is inhibited as the fluid flow is not forced from the secondary finger into the primary finger, meaning much greater interaction and non-linear dynamics are seen between growing fingers.

\section{Long time scale evolution}

To investigate the complex interfacial dynamics and non-linear growth that occurs in a realistic injection case, asymmetry can be introduced into the starting perturbed bubble interface. By including different wavelengths of perturbation along the interface, multiple length scales are produced, mimicking that which would be found in reality due to random noise and disturbance. Long time evolutions of the interface are presented in this section, in order to see the effect of finger interaction when shielding is inhibited. 


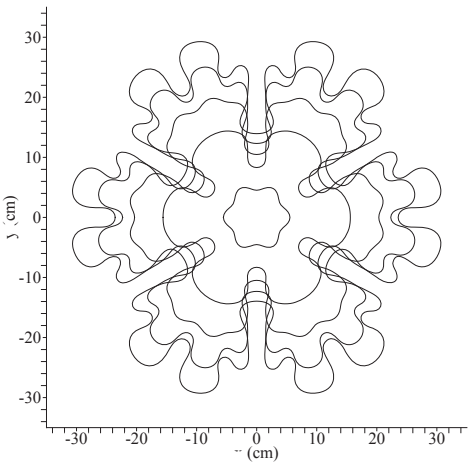

(a) $\beta=10$

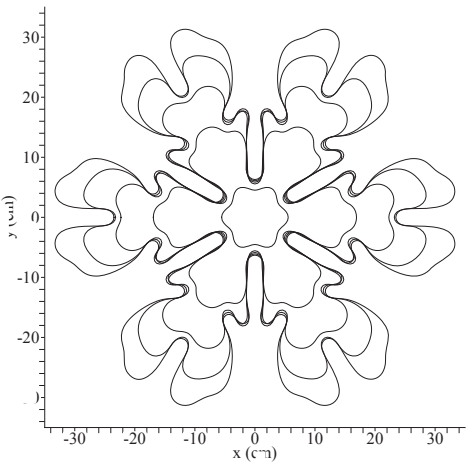

(b) $\beta=1000$

Figure 3: Bubble evolution plots with varying mobility ratio, $\beta$. Each subplot shows the interface of the bubble every 100 s from $0-400$ s.

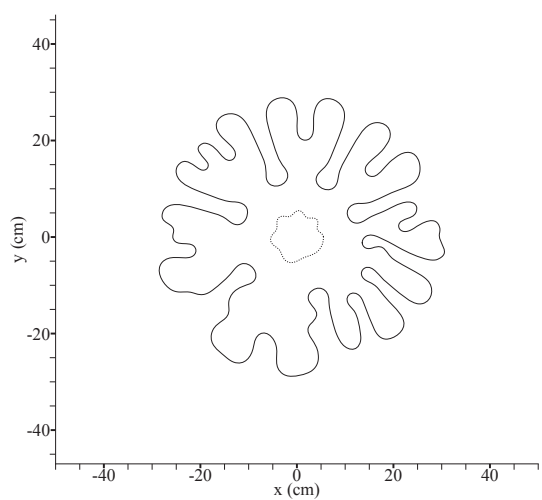

(a) $t=350 \mathrm{~s}$

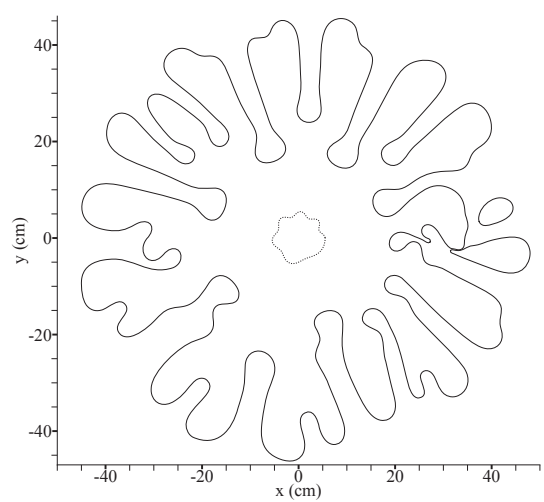

(b) $\mathrm{t}=850 \mathrm{~s}$

Figure 4: Bubble Interface of asymmetric $\mathrm{CO}_{2}(\mathrm{sc})$ injection at different times. Dotted interface shows the initial bubble profile.

Presented in figure 4 is a long time interface evolution using parameters defined for a realistic supercritical $\mathrm{CO}_{2}$ injection, with $\mathrm{Ca}^{\prime}=60.5$ and $\beta=10.86$.

The bases of the fingers in figure 4 have moved significantly from the starting profile and have not formed stagnation points, as usually found in high mobility ratio flows. The smallest wavelength fingers on the right of the domain have a large interaction with each other, with severe base thinning occurring at two different locations. Due to the relatively large capillary number, the critical length scale of bifurcation is small, allowing side branching to form on some of the larger fingers. Competing fingers can grow very close to each other, creating a small immiscible lubrication layer between them. 
Figure 4b shows two disjoint bubbles of $\mathrm{CO}_{2}$ that have detached from the main plume due to base thinning on the primary finger. The first finger to detach is caused by the primary finger growing behind it, however the second finger breakoff is due to a combination of thinning caused by fingers growing into the left and right side of it. After the second break-off, the finger that is still attached to the main plume acts to push the trailing edge of the detached bubble, which along with surface tension quickly forms it to a droplet shape. The small attached finger left from the second break-off quickly recedes due to the very high curvature and re-stabilising effect of surface tension.

This kind of breaking has been reported before in [9] and [10], however, using a diffuse interface model or volume tracking technique does not as accurately resolve the interface between the fluids as an interface tracking method. Interface capturing introduces a level of uncertainty about the exact position of the interface and hence whether breaking or coalescence should occur when two fingers grow very close to each other. [7] and [8] showed experimental that coalescing and finger break-off could occur, although a small miscible region exists between the fluids experimentally, as the liquids are never completely immiscible.

In the present model, if a sufficiently high enough element density is used, finger break-off always occurs in preference to coalescence. The fluids are considered to be completely immiscible and therefore no coalescence should occur, as a lubrication layer should always separate the fingers.

\section{Conclusion}

A two-phase BEM formulation for solving low mobility ratio flows has been developed and used to investigate viscous fingering mechanisms using realistic $\mathrm{CO}_{2}$ sequestration fluid properties. When the mobility ratio of the two fluids is of order $10-50$, the fingering characteristics are vastly different to those predicted by single-phase, or high mobility ratio models. The near stagnation points on the bases of the fingers found in single-phase flows were not found when using the two-phase model for low mobility ratio flows.

Finger interaction was found to be much more significant than in single-phase models, and on small wavelength perturbations could lead to base thinning and eventual finger breaking. After breaking, the detached bubbles would continue with the velocity of the surrounding fluid.

\section{References}

[1] Saffman, P. \& Taylor, G., The penetration of a fluid into a porous medium or Hele-Shaw cell containing a more viscous liquid. Proceedings of the Royal Society of London Series A: Mathematical and Physical Sciences, 245(1242), pp. 312-329, 1958.

[2] Homsy, G., Viscous fingering in porous media. Annual Review of Fluid Mechanics, 19, pp. 271-311, 1987. 
[3] DeGregoria, A. \& Shwartz, L., A boundary-integral method for two-phase displacement in hele-shaw cells. Journal of Fluid Mechanics, 164, pp. 383400, 1986.

[4] Li, S., Lowengrub, J. \& Leo, P., A rescaling scheme with application to the long-time simulation of viscous fingering in a Hele-Shaw cell. Journal of Computational Physics, 25(1), pp. 554-567, 2007.

[5] Hadavinia, H., Advani, S. \& Fenner, R., The evolution of radial fingering in a Hele-Shaw cell using $C^{1}$ continuous Overhauser boundary element method. Engineering Analysis with Boundary Elements, 16, pp. 183-195, 1995.

[6] Hansen, E., A numerical study of unstable Hele-Shaw flow. Computers and Mathematics with Applications, 38, pp. 217-230, 1999.

[7] Moore, M., Juel, A., Burgess, J., McCormick, W. \& Swinney, H., Fluctuations and pinch-offs observed in viscous fingering. Proceedings of the Seventh Experimental Chaos Conference, Springer-Verlag, pp. 189-194, 2003.

[8] MacMinn, C., Szulczewski, M., Cueto-Felgueroso, L. \& Juanes, R., Experimental characterization of immiscible viscous fingering in a HeleShaw cell. American Geophysical Union Meeting, 2009.

[9] Sun, Y. \& Beckermann, C., A two-phase diffuse-interface model for HeleShaw flows with large property contrasts. Physica D, 237, pp. 3089-3098, 2008.

[10] Guan, X. \& Pitchumani, R., Viscous fingering in a hele-shaw cell with finite viscosity ratio and interfacial tension. Journal of Fluids Engineering, 125, pp. 354-363, 2003.

[11] Power, H. \& Wrobel, L., Boundary Integral Methods in Fluid Mechanics. Computational Mechanics Publications, Southampton, 1995.

[12] Power, H., The evolution of radial fingers at the interface between two viscous liquids. Engineering Analysis with Boundary Elements, 14(4), pp. 297-304, 1994.

[13] Jawson, M. \& Symm, G., Integral Equations Methods in Potential Theory and Elastostatics. Academic Press, New York, 1977.

[14] Batchelor, G., An Introduction to Fluid Dynamics. Cambridge University Press, 1967.

[15] Cabral, J., Wrobel, L. \& Brebbia, C., A BEM formulation using B-splines: I Uniform blending functions. Engineering Analysis with Boundary Elements, 7(3), pp. 136-144, 1990.

[16] Guigianni, M., Formulation and numerical treatment of boundary integral equations with hypersingular kernels. Singular Integrals in Boundary Element Methods, eds. V. Sladek \& J. Sladek, Computational Mechanics Publications, pp. 85-125, 1998.

[17] Ouyang, L., New correlations for predicting the density and viscosity of supercritical carbon dioxide under conditions expected in carbon capture and sequestration operations. The Open Petroleum Engineering Journal, 4, pp. 13-21, 2011. 